\title{
Immunotherapy in Merkel cell carcinoma: role of Avelumab
}

\section{Amruth R Palla \\ Donald Doll}

Department of Internal Medicine, Division of Hematology and Oncology, Ellis Fischel Cancer Center, University of Missouri, Columbia, MO, USA

Correspondence: Amruth R Palla Department of Internal Medicine, Division of Hematology and Oncology, Ellis Fischel Cancer Center, University of Missouri, I Hospital Drive, Columbia, MO 652।2, USA

Tel +I $5738824 \mid 4$ I

Email pallaa@health.missouri.edu
This article was published in the following Dove Press journal: ImmunoTargets and Therapy

\begin{abstract}
Merkel cell carcinoma (MCC), a rare skin cancer, is associated with high mortality, especially in a metastatic setting. Though conventional chemotherapy with platinum and etoposide has had high response rates, many of the patients have had early relapse without any effective therapy thereafter. Recently, immune check point inhibitors have shown very good durable responses, leading to the approval of a programmed death-ligand 1 inhibitor Avelumab for these patients. We briefly review the epidemiology and immune basis of the pathogenesis of MCC, which therefore explains the excellent response to check point inhibitors, and throw light on future directions of immunotherapy for this cancer.
\end{abstract}

Keywords: Merkel cell carcinoma, T cell, PD-L1, Avelumab, immunotherapy, check point inhibitors, neuroendocrine tumor

\section{Introduction}

Merkel cell carcinoma (MCC) is a very rare skin cancer affecting about 1500-2000 people per year in the United States and its incidence is on the rise. Though rare, it has a much higher rate of mortality (about 15\% more) compared to the more common cutaneous melanoma. ${ }^{1}$ It is thought to arise from the clear oval cells in the stratum basale of the epidermis which are also called Merkel cells, to honor Friedrich Merkel who had first described them in 1875. Though the embryonal origin of the Merkel cells from epidermal versus neural crest cells has been an area of controversy for a long time, recent data strongly suggest an epidermal origin for the mammalian Merkel cells. A majority of the Merkel cells are in contact with nerve endings that receive the sensation of touch. However, a minority are devoid of this contact with the nerve endings when they form part of the neuroendocrine system that modulates peripheral neural responses. Merkel cell carcinoma is mainly thought to arise from the latter than the former type of Merkel cells. ${ }^{2}$ Given this nature of the Merkel cells, this cancer has also been labeled as the "neuroendocrine cancer of the skin". Indeed, on immunohistochemistry Merkel cells can be detected by antibodies against neuroendocrine markers such as chromogranin and synaptophysin.

Etiology for MCC includes ultraviolet (UV) light, immunosuppression, and infection with the polyomavirus. ${ }^{1}$ Merkel polyomavirus (MPV) is implicated in close to $80 \%$ of these cancers. ${ }^{1}$ MCC generally arises in areas of higher sun (near the equator) and in people with lighter skin pigmentation (Caucasians). ${ }^{1}$ It generally presents in sun exposed areas such as the face and extremities. ${ }^{1}$ Only $10-15 \%$ of the cases arise in nonsun exposed areas such as trunk, buttocks or genitalia. ${ }^{1}$ It generally occurs in older people over the age of 50 years and the incidence increases with advancing age. ${ }^{1}$ 
Grossly, the tumor appears as a painless, firm, raised growth that has the same color as the skin or can be red or violet. ${ }^{1}$ As mentioned previously antibodies against neuroendocrine markers are commonly used to detect the cancer cells on immunohistochemistry. Cytokeratin (CK) 20 is another marker to detect these cells and the cancer is typically positive for CK-20 and negative for CK-7, though rare cases with positive $\mathrm{CK}-7$ have been reported. ${ }^{3}$

MCC shows predisposition for relatively rapid growth and metastasis. It commonly metastasizes to local lymph nodes before spreading to distant lymph nodes, skin or other organs. It is not uncommon for it to be detected in lymph nodes without a known primary site. ${ }^{1}$ TNM system is used for staging with earlier stages having smaller sized tumors and no lymph node involvement, and large tumors with lymph node involvement and distant metastases comprising the later stages. ${ }^{1}$

Treatment for localized MCC includes surgery with or without radiation therapy. The cure rates are as high as $95 \%$, but more than half of these patients later have recurrence, commonly with distant disease. Metastatic MCC has conventionally been treated akin to neuroendocrine small cell cancer of the lung, with platinum and etoposide chemotherapy. Though the response rates to such therapy may be as high as $66 \%,>50 \%$ of patients have recurrence within the first 3 months after completion of chemotherapy. Until recently, there were no effective second-line therapies available for patients with metastatic MCC who had recurrence after first-line chemotherapy and the prognosis was dismal. Thus, clinicians and researchers are enthusiastically investigating novel therapies for this population. Currently available data from check point inhibitor therapy in the metastatic setting looks very promising and results of further ongoing clinical trials incorporating these agents in both early and advanced/ metastatic settings are eagerly awaited.

\section{Role of T cell impairment in the occurrence of MCC and its prognosis}

Various epidemiologic and clinical research has proven the importance of pathologic variation in either quantitative or qualitative norm of $\mathrm{T}$ cells in the occurrence and prognosis of MCC.

\section{Increased incidence of MCC in immunosuppressed patients}

Around 5-50\% increased incidence has been noted by various authors in various categories of immunosuppressed patient populations including HIV positive patients, ${ }^{4}$ solid organ transplant recipients on immunosuppressive therapies, ${ }^{5}$ and in patients with B cell malignancies with suppressed $\mathrm{T}$ cell function (for example, chronic lymphocytic leukemia). ${ }^{6}$

\section{Increased incidence of MCC in older people}

MCC is extremely rare before the age of 50 and its incidence continues to increase beyond age 50. It is a well-known fact that there is some lymphopenia and physiologic qualitative immunodeficiency with aging, which is postulated to be the reason for this incidence curve in patients with MCC. ${ }^{1}$

\section{Decreased survival observed at a given TNM stage of MCC for immunosufficient versus immunodeficient patients}

Improved survival has been observed in MCC patients who were found to have cancer in lymph nodes with an unknown primary, compared to those with cancer in lymph nodes and a known primary. ${ }^{7,8}$ In fact, the survival was noted to be almost doubled in the former group compared to the latter. It is believed that patients with a well-functioning immune system may successfully eliminate their primary and thus have an unknown primary at the time of presentation. Likewise, patients with a poorly functioning immune system may not eliminate their primary and hence present with a known primary. This was demonstrated in a study by Asgari et al, ${ }^{9}$ in a cohort of patients with stage IIIB MCC, consisting of patients with immunosuppression and those with preserved immunity. None of the patients who were immunosuppressed could eliminate their primary and thus $100 \%$ of them presented with a primary, compared to the group with preserved immunity, in whom $66 \%$ of patients presented with an unknown primary. ${ }^{9}$

Paulson et al reported a proportionate improvement in survival of patients with MCC in comparison to the increasing percentage of normally functioning CD8 tumor infiltrating lymphocytes in their tumors. ${ }^{10}$

\section{Role of MPV}

Oncogenic viruses impairing $\mathrm{T}$ cell mediated immunity leading to occurrence of cancer has been well recognized in recent years. MPV infected MCC tumors have been shown to have increased dysregulated tumor infiltrating lymphocytes with downregulated major histocompatibility complex-1 receptor binding T cell receptors. ${ }^{11}$

Such findings suggest that the basis of the pathogenesis in the majority of the cases of MCC is immunogenic, and thus 
provide a rationale for using treatments that can intervene at various interaction points of T lymphocytes and tumor cells.

\section{Physiology of T lymphocyte interaction with the tumor cell}

After recognizing the importance and influence of immune mediated pathogenesis in the development and the clinical course of MCC, it was necessary to understand the physiology of the interaction of T lymphocytes with the tumor cells, to identify potential targets against which molecules could be developed to enhance T-lymphocyte mediated destruction of the tumor cells.

Research studies have identified the foci of interaction between $\mathrm{T}$ lymphocytes and tumor cells (antigen presenting cells). Tumor cells infected with the MPV or those that underwent UV radiation induced mutations serve as the antigen presenting cells. Some of these interactions result in stimulation of the T cells to effector T cells, which then destroy the tumor cells. However, it has been noted that tumor cells may develop their own mechanisms to evade this immune attack. Tumor cells express specific proteins on their cell surface, which interact with the complementary proteins on the $\mathrm{T}$ cell surface, resulting in inactivation of T cells which then become dysfunctional and eventually undergo apoptosis. ${ }^{12}$

Table 1 summarizes some of the important interactions of $\mathrm{T}$ lymphocyte surface proteins with tumor cell surface proteins, the effect of these interactions on the T lymphocyte and targets against which drugs have been developed.

Avelumab-a programmed death-ligand 1 (PD-L1) inhibitor, Pembrolizumab and Nivolumab, both programmed death-1 (PD-1) inhibitors, are now included in the National Comprehensive Cancer Network (NCCN) guidelines for the treatment of patients with metastatic MCC, both in the first line or in the relapsed refractory setting. ${ }^{13}$

Currently, Avelumab remains the only check point inhibitor that is US Food and Drug Administration (FDA) approved for this indication.

\section{Role of Avelumab in the treatment of MCC}

Avelumab is a PD-L1 inhibitor which blocks human IgG1 lambda monoclonal antibody on the tumor cell, inhibits the interaction between the PD-1 on T lymphocyte with the PD-L1 on the tumor cell, thus preventing the inactivation of the T lymphocyte and keeping it available for tumor cell destruction.

On March 23, 2017, Avelumab (BAVENCIO; EMD Serono, Inc.), received accelerated approval by the FDA for the treatment of metastatic MCC in the relapsed/refractory setting for patients above 12 years of age. This approval was based on the Phase II, multicenter, international, prospective, single group, open label trial - the "Javelin Merkel 200 trial". ${ }^{14}$ In this trial patients aged 18 years or older, with histologic confirmation of MCC while on, or after cytotoxic chemotherapy, with an Eastern Cooperative Oncology Group performance status of 0 or 1 , measurable disease by Response Evaluation Criteria in Solid Tumors (RECIST) version 1.1, adequate hepatic, and renal and hematologic reserve, and immune-competent status were enrolled from 35 international cancer treatment centers. Patients with HIV, immunosuppression, hematological malignancies, and previous organ transplantation were excluded. Merkel cell polyomavirus or PD-L1 expression status were not used in selecting patients for the study. A total of 88 patients were

Table I Some important interactions of T lymphocyte surface proteins with tumor cell surface proteins

\begin{tabular}{|c|c|c|c|}
\hline $\begin{array}{l}\text { T lymphocyte surface } \\
\text { protein }\end{array}$ & $\begin{array}{l}\text { Tumor cell surface protein } \\
\text { with which this } \mathrm{T} \text { lymphocyte } \\
\text { protein interacts }\end{array}$ & $\begin{array}{l}\text { Effect of this interaction } \\
\text { on the } T \text { cell }\end{array}$ & Comment \\
\hline T cell receptor & $\mathrm{MHC}$ & Stimulation & $\begin{array}{l}\text { First step in the activation of } T \text { cells to effector } \\
T \text { cells }\end{array}$ \\
\hline CD28 & B 7.1 and B 7.2 & Co-stimulation & $\begin{array}{l}\text { Second step in the activation of } T \text { cells to } \\
\text { effector } T \text { cells }\end{array}$ \\
\hline $\begin{array}{l}\text { CTLA4 } \\
\text { (Cytotoxic T lymphocyte } \\
\text { antigen 4) }\end{array}$ & B 7.1 and B 7.2 & $\begin{array}{l}\text { Prevention of } \\
\text { co-stimulation } \\
\text { (by blocking the } \\
\text { B } 7 \text { proteins from interacting } \\
\text { with CD28 proteins) }\end{array}$ & $\begin{array}{l}\text { CTLA4 is the target for Ipilimumab, which } \\
\text { blocks CTLA } 4 \text { and thus allows the uninhibited } \\
\text { interaction of CD28 with B } 7 \text { molecules } \\
\text { leading to co-stimulation of T cells }\end{array}$ \\
\hline $\begin{array}{l}\text { PD-I } \\
\text { (Programmed death-I) }\end{array}$ & $\begin{array}{l}\text { PD-LI } \\
\text { (Programmed death-ligand I) }\end{array}$ & Inactivation & $\begin{array}{l}\text { PD-I and PD-LI are targets for check point } \\
\text { inhibitors which prevent the interaction } \\
\text { of PD-I with PD-LI and thus prevent the } \\
\text { inactivation of T cells }\end{array}$ \\
\hline
\end{tabular}

Notes: Data from Preusser et al. ${ }^{17}$ 
enrolled, and all these patients received at least one dose of Avelumab which was given intravenously at a dose of $10 \mathrm{mg} / \mathrm{kg}$ every 2 weeks. Overall response rate (ORR) (either complete or partial response), which was the primary end point, was assessed by an independent review committee as per the RECIST version 1.1. Of the 88 patients enrolled, 29 patients achieved a response -10 patients (11\%) with complete response (CR) and 19 patients (22\%) with partial response (PR). The ORR was 33\% (95\% confidence interval [CI]: 23.3-43.8). The response duration ranged from 2.8 to $23.3+$ months with $86 \%$ of responses durable for 6 months or longer. Responses were seen regardless of PD-L1 tumor expression or presence of Merkel cell polyomavirus. The most common adverse reactions observed were fatigue, musculoskeletal pain, diarrhea, nausea, infusion-related reaction, rash, decreased appetite, and peripheral edema. Serious adverse reactions that occurred in more than one patient in the trial were acute kidney injury, anemia, abdominal pain, ileus, asthenia, and cellulitis.

A 1-year efficacy update of the above trial was presented recently by Kaufman et al at the American Association for Cancer Research. ${ }^{15}$ In the interim period (as of September $3,2016)$ treatment was ongoing in $22 \%(n=19)$. The main reasons for discontinuations were disease progression $(n=44$; $50 \%)$, death $(n=7 ; 8 \%)$, adverse event $(n=7 ; 8 \%)$, or withdrawal $(n=4 ; 5 \%)$. Two more responses were noted from the initial 6 month analysis. Of these two, one was a new $\mathrm{CR}$ and another patient had improved from PR to CR. Responses were ongoing in $21 / 29$ patients $(72.4 \%)$ at the time of analysis. The estimated proportion of responders with $\geq 1$-year duration of response was 74\% (95\% CI 53-87). Estimated 1-year progression-free survival rate was 30\% (95\% CI 21-41) and 1-year overall survival (OS) rate was 52\% (95\% CI 41-62). Median OS was 12.9 months (95\% CI 7.5-not estimable). These data suggested long-term benefit with Avelumab in a proportion of patients with relapsed/refractory metastatic MCC.

The high ORR and the prolonged durable responses noted with Avelumab, at least in a proportion of patients with metastatic MCC who were refractory to or who relapsed after cytotoxic chemotherapy, are very encouraging, especially given the otherwise grim prognosis in this population of patients prior to check point inhibitor therapies.

There are no studies directly comparing the efficacy of Avelumab to established chemotherapy with platinum and etoposide in this patient population, and most possibly such studies will never be conducted given the durable responses seen with check point inhibitor therapy as compared to the short-term responses seen with chemotherapy. We believe that in the present-day chemotherapy will most likely be reserved for metastatic MCC patients with high tumor burden and acute tumor related symptoms where a faster response is desired. It can also remain an option in patients who progress on frontline treatment with check point inhibitors but still have a decent performance status and desire some kind of second-line therapy.

\section{Future directions with Avelumab and other check point inhibitors in MCC}

Currently, though Avelumab has accelerated approval by FDA only for the relapsed/refractory setting, National Comprehensive Cancer Network recommends using it even as first-line treatment in patients with metastatic MCC. Also, as previously mentioned, both Pembrolizumab and Nivolumab are included for this indication, under National Comprehensive Cancer Network guidelines. Nghiem et al conducted a Phase II, multicentric, noncontrolled study of frontline Pembrolizumab in patients with advanced MCC and they reported an ORR of $56 \%$, in patients with and without polyomavirus positive MCC. ${ }^{16}$ In this study, Pembrolizumab was administered at a dose of $2 \mathrm{mg} / \mathrm{kg}$ of body weight every 3 weeks. It would not be unreasonable to anticipate further FDA approvals of Avelumab and other check point inhibitors in frontline therapy for patients with advanced MCC, in the near future.

Trials using Avelumab in the adjuvant setting in MCC are also ongoing (ClinicalTrials.gov ID: NCT03271372) as are trials using other check point inhibitors, Nivolumab in adjuvant MCC (ClinicalTrials.gov ID: NCT02196961) and Nivolumab and Ipilimumab, with or without stereotactic body radiation therapy, in treating patients with recurrent or stage IV MCC (Clinicaltrials.gov ID: NCT03071406).

Overall, the future with check point inhibitor therapies either as single agents or in combination with other agents looks very promising for patients with MCC.

\section{Disclosure}

The authors report no conflicts of interest in this work.

\section{References}

1. Becker JC, Stang A, Hausen AZ, et al. Epidemiology, biology and therapy of Merkel cell carcinoma: conclusions from the EU project IMMOMEC. Cancer Immunol Immunother. Epub 2017 Nov 30.

2. Van Keymeulen A, Mascre G, Youseff KK, et al. Epidermal progenitors give rise to Merkel cells during embryonic development and adult homeostasis. J Cell Biol. 2009;187(1):91-100.

3. Khader A, George S, Balakrishnan S, Aravindan KP. Spontaneous regression in an ulcerated CK7 positive Merkel cell carcinoma. Indian J Dermatol Venereol Leprol. 2015;81(2):170-173. 
4. Fukumoto H, Sato Y, Hasegawa H, Katano H. Frequent detection of Merkel cell polyomavirus DNA in sera of HIV-1-positive patients. Virol J. 2013;10:84.

5. Sparks J, Sparks M, Malone JC. Cutaneous Merkel cell carcinoma: multiple asynchronous primary lesions in a patient on immunosuppressive therapy. J Cutan Pathol. 2017;44(3):309-312.

6. Velez NF, Karia PS, Vartanov AR, Davids MS, Brown JR, Schmults CD. Association of advanced leukemic stage and skin cancer tumor stage with poor skin cancer outcomes in patients with chronic lymphocytic leukemia. JAMA Dermatol. 2014;150(3):280-287.

7. Arron ST, Canavan T, Yu SS. Organ transplant recipients with Merkel cell carcinoma have reduced progression-free, overall, and diseasespecific survival independent of stage at presentation. J Am Acad Dermatol. 2014;71(4):684-690.

8. Paulson KG, Iyer JG, Blom A, et al. Systemic immune suppression predicts diminished Merkel cell carcinoma-specific survival independent of stage. J Invest Dermatol. 2013;133(3):642-646.

9. Asgari MM, Sokil MM, Warton EM, Iyer J, Paulson KG, Nghiem P. Effect of host, tumor, diagnostic, and treatment variables on outcomes in a large cohort with Merkel cell carcinoma. JAMA Dermatol. 2014;150(7):716-723.

10. Paulson KG, Iyer JG, Tegeder AR, et al. Transcriptome-wide studies of merkel cell carcinoma and validation of intratumoral CD8+ lymphocyte invasion as an independent predictor of survival. J Clin Oncol. 2011;29(12):1539-1546.
11. Moens U, Rasheed K, Abdulsalam I, Sveinbjørnsson B. The role of Merkel cell polyomavirus and other human polyomaviruses in emerging hallmarks of cancer. Viruses. 2015;7(4):1871-1901.

12. Carter LL, Carreno BM. Cytotoxic T-lymphocyte antigen-4 and programmed death-1 function as negative regulators of lymphocyte activation. Immunol Res. 2003;28(1):49-59.

13. National Comprehensive Care Network (NCCN). Merkel Cell Carcinoma Guidelines-Systemic therapy guidelines. Available from: https:// www.nccn.org/professionals/physician_gls/pdf/mcc.pdf. Accessed February 23rd, 2018.

14. Kaufman HL, Russell J, Hamid O, et al. Avelumab in patients with chemotherapy-refractory metastatic Merkel cell carcinoma: a multicentre, single-group, open-label, phase 2 trial. Lancet Oncol. 2016;17(10):1374-1385.

15. Kaufman HL, Russell JS, Hamid O, et al. CT079: Durable responses to avelumab (anti-PD-L1) in patients with Merkel cell carcinoma progressed after chemotherapy: 1-year efficacy update. Presented at: AACR Annual Meeting 2017; April 3, 2017; Washington, DC.

16. Nghiem PT, Bhatia S, Lipson EJ, et al. PD-1 blockade with pembrolizumab in advanced Merkel-cell carcinoma. NEngl J Med. 2016;374(26): 2542-2552.

17. Preusser M, Berghoff AS, Thallinger C, Zielinski CC. Cancer immune cycle: a video introduction to the interaction between cancer and the immune system. ESMO Open. 2016;1(3):e000056. eCollection 2016.
ImmunoTargets and Therapy

\section{Publish your work in this journal}

ImmunoTargets and Therapy is an international, peer-reviewed open access journal focusing on the immunological basis of diseases, potential targets for immune based therapy and treatment protocols employed to improve patient management Basic immunology and physiology of the immune system in health, and disease will be also covered. In addition, the journal will focus on the impact of manage-

\section{Dovepress}

ment programs and new therapeutic agents and protocols on patient perspectives such as quality of life, adherence and satisfaction. The manuscript management system is completely online and includes a very quick and fair peer-review system, which is all easy to use. Visit http://www.dovepress.com/testimonials.php to read real quotes from published authors. 